\title{
Physically-Based Droplet Interaction
}

\author{
Richard Jones \\ Centre for Digital Entertainment \\ Double Negative \\ richard.jones@bournemouth.ac.uk
}

\author{
Richard Southern \\ Centre for Digital Entertainment \\ National Centre for Computer Animation \\ rsouthern@bournemouth.ac.uk
}

\begin{abstract}
In this paper we present a physically-based model for simulating realistic interactions between liquid droplets in an efficient manner. Our particle-based system recreates the coalescence, separation and fragmentation interactions that occur between colliding liquid droplets and allows systems of droplets to be meaningfully represented by an equivalent number of simulated particles. By considering the interactions specific to liquid droplet phenomena directly, we display novel levels of detail that cannot be captured using other interaction models at a similar scale. Our work combines experimentally validated components, originating in engineering, with a collection of novel modifications to create a particle-based interaction model for use in the development of mid-to-large scale dropletbased liquid spray effects. We demonstrate this model, alongside a size-dependent drag force, as an extension to a commonly-used ballistic particle system and show how the introduction of these interactions improves the quality and variety of results possible in recreating liquid droplets and sprays, even using these otherwise simple systems.
\end{abstract}

\section{CCS CONCEPTS}

- Computing methodologies $\rightarrow$ Physical simulation;

\section{KEYWORDS}

Fluid simulation, Particle systems

\section{ACM Reference format:}

Richard Jones and Richard Southern. 2017. Physically-Based Droplet Interaction. In Proceedings of SCA '17, Los Angeles, CA, USA, fuly 28-30, 2017, 10 pages.

DOI: $10.1145 / 3099564.3099573$

\section{INTRODUCTION}

Ballistic particle systems are a key component in the creation of simulated liquid effects, commonly used to quickly model the motion of airborne liquid droplets/spray and so add finer-scale details to free-surface liquid simulations. These are heavily used in animation and visual effects production[Froemling et al. 2007; Iversen and Sakaguchi 2004; Kurtz and Duda 2002], and their application is also well documented in technical works including coupled liquid spray and free-surface models[Kim et al. 2006; Mihalef et al. 2009; Song et al. 2005; Takahashi et al. 2003; Yang et al. 2014] and purely whitewater-style simulations[Ihmsen et al. 2012]. These systems often ignore internal interaction entirely, assume interactions only

SCA '17, Los Angeles, CA, USA

(C) 2017 ACM. This is the author's version of the work. It is posted here for your personal use. Not for redistribution. The definitive Version of Record was published in Proceedings of SCA '17, fuly 28-30, 2017, https://doi.org/10.1145/3099564.3099573. result in droplet merging, or rely on coupling to larger-scale fluid simulation (and as such are restricted to its resolution) to give any notion of inter-droplet interaction. In this way, high quality results are very difficult to create without considerable user input. In particular, no consideration has been given to the full range of realistic interactions that occur between droplets such as separating and fragmenting phenomena. However, these interactions are known to be key influences on the size and velocity distributions within droplet sprays[Kim et al. 2009].

We develop a system to incorporate these missing interactions through the physically-based modelling of droplet collisions and their outcomes. This added interaction increases the quality of particle-based spray simulations and so reduces existing requirements on user input to create realistic results. By modelling droplet collisions directly we introduce physically-plausible interactions whilst avoiding the drastic increase of complexity that would be required to capture these phenomena using traditional free-surface or multi-phase liquid simulation methods.

Droplet collisions are a key factor in the behaviour of liquid sprays that have until now been largely ignored by the graphics community. Introducing these phenomena into a ballistic particle system allows the particles to meaningfully represent droplets ${ }^{1}$ and recreate small-scale interactions. Modelling droplet collisions is well-studied and documented in the fields of engineering and meteorology, where it is used to alleviate requirements for high resolution multi-phase simulation to simulate internal interaction in liquid sprays[Kim et al. 2009; O'Rourke 1981]. A common approach is to predict and resolve a set of observed outcomes of collisions between pairs of droplets i.e. coalescence and separation (Fig. 1) using discrete threshold methods[Ashgriz and Poo 1990; Brazier-Smith et al. 1972]. These methods have been shown to efficiently simulate realistic interactions between large numbers of droplets within a liquid spray and are therefore able to quickly and easily create meaningful recreations of these phenomena. Furthermore, these methods facilitate the incorporation of models that define physically-based fragmentation and breakup of droplets[Munnannur and Reitz 2007], creating mechanisms for droplet emission to occur naturally throughout our simulations and so add finer scales of detail to the results. For our application in computer graphics, we combine these components into a system to model droplets and their interactions on a large scale, enabling increased quality of results for millions of small, yet visible, droplets.

The contributions of this paper can be summarised as:

\footnotetext{
${ }^{1}$ In computer graphics, the scale of a 'droplet' is usually somewhat different to that in engineering references $(O(\mu \mathrm{m}))$ but in this paper, we use the realistic collision behaviours of droplets, and their motivating physical interactions, as an approximation of the interactions that should occur between our often larger liquid 'droplets'. We consider droplets to be any visible isolated form of water that can be reasonably approximated by a sphere.
} 
- The introduction of the coalescence, separating and fragmenting collision outcomes into particle-based simulations of liquid droplets for computer graphics.

- A novel particle interaction model that combines physicallybased outcome determination, trajectory-based binary collision detection and non-uniform fragmentation rules to recreate droplet phenomena for graphics applications.

- The application of this model to a simple ballistic particle system for liquid droplets and spray, creating efficient particle simulations that move under the effects of gravity, drag and plausible droplet interaction.

\section{BACKGROUND}

For an overview of the models of liquid sprays used in engineering, we direct the reader to the work of Sirignano [2010], and those interested in further information in droplet modelling specifically, the book by Ashgriz [2011]. Here we focus on the previous works within computer graphics that tackle simulations of spray, droplets and similar liquid phenomena.

\subsection{Related work}

Fine-scale, high detail splash and spray features are hard to capture with most incompressible liquid simulation frameworks and as such, secondary particle systems have long been used in computer graphics to increase effective resolution and create missing features. As these phenomena do not exhibit standard single-phase incompressible fluid characteristics (at the same scale as the main simulations, at least), motion is often assumed to be simply ballistic (or sometimes with some notion of drag).

Following this basic framework, many previous works have focused on the emission of these ballistic particles. Methods based around Eulerian liquid simulation techniques include: emission of ballistic particles from areas of high curvature of the surface in a VOF simulation[Takahashi et al. 2003]; a mass preserving two-way coupling of a level-set liquid and droplet/bubble particles[Song et al. 2005]; and the emission of ballistic droplets and/or bubbles from erroneous free-surface tracking particles[Kim et al. 2006; Mihalef et al. 2009]. Ihmsen et al. [2012] introduce a framework for spray, bubble and foam particles, generated from an initial Lagrangian particle-based liquid simulation. This work uses a decoupled ballistic particle simulation to create whitewater elements following an initial free-surface simulation. This decoupled approach is commonly used in visual effects due to its flexibility, as it removes the requirement for full liquid re-simulation when making even minor changes to the result[Froemling et al. 2007; Iversen and Sakaguchi 2004; Kurtz and Duda 2002]. In this paper, we demonstrate the addition of droplet interaction into a simple stand-alone particle system, but our model could be used with any of the above emission frameworks.

Moving away from purely ballistic motion, Losasso et al. [2008] and Yang et al. [2014] look to incorporate fluid-like interaction into these secondary particle systems by blending between ballistic and fluid motion (from a background grid) based on local particle density. Other work from Chentanez et al. [2015] uses a similar blend between different models based on distance to the fluid surface, but instead use Lagrangian particle fluid methods such as PBF[Macklin

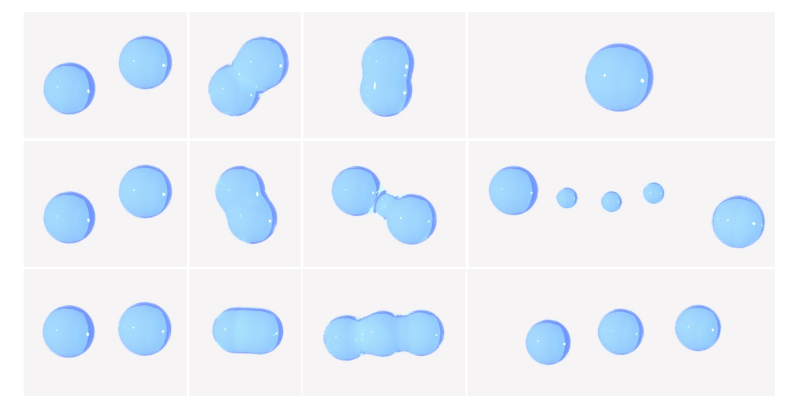

Figure 1: Binary droplet interactions (start - left, end - right): coalescence (top) - $\mathrm{We}=16$, stretching separation (middle) $\mathrm{We}=447$ and reflexive separation (bottom) $-\mathrm{We}=155$, simulated with our particle-based collision model.

and Müller 2013] or SPH[Ihmsen et al. 2014] for the spray components. Whilst these blending models offer more plausible spray motion in areas of higher particle density, interaction in areas of low density continues to be ignored (ballistic), not properly-defined (e.g. SPH in low density - Fig.7), or restricted by the grid resolution of the underlying fluid velocity grid. Our work addresses these problems by directly modelling each particle as a droplet in a meaningful way, creating realistic behaviours at a finer scale than handled by these approaches, whilst maintaining similar resolutions.

Instead of a particle-based approach to liquid sprays, Nielsen and Østerby [2013] develop a physically-based model for volumetric sprays using a two-continua (air/liquid) discretisation. They share our motivation of utilising a more physically-motivated representation of sprays, but consider a macroscopic, volumetric approximation with a focus on motion with respect to the surrounding simulated air. As such, they are unable to recreate individual droplet details. Our method aims to create higher levels of detail in particlebased spray simulations specifically, to help better replicate sprays of visible droplets and their interactions. We forsee that a combination of our smaller-scale direct interactions and larger-scale indirect interactions[Losasso et al. 2008; Nielsen and Østerby 2013; Yang et al. 2014] could be the foundation of a more physically-motivated multi-scale method in the future, but feel this lies beyond the scope of a single paper.

Other models considering droplet phenomena can be found in the work on droplet and liquid flow on solid objects from Wang et al. [2005], using signed distance functions, and Azencot et al. [2015], using meshes. Also, with similar motivation to ours for droplets, interactions between other small-scale multi-phase phenomena have been considered, such as bubbles [Busaryev et al. 2012; Patkar et al. 2013].

Finally, high quality simulations of small-scale liquid phenomena (including droplet interactions) have been shown by Da et al. [2016]. This work considers surface-tension dominated liquid surface phenomena as we do, but with a focus on high quality tracking of deforming liquid surfaces at this small-scale, and so incurs much greater computational cost. We instead choose to approximate a collection of similar behaviours using a particle-based method, allowing application to systems of millions of droplets with relative ease, as well as integration into existing simulation frameworks. 


\subsection{Droplet interactions}

In reality, droplets within liquid sprays directly interact through collisions that can cause them to deform, merge and break-up. These localised interactions greatly affect the velocity and size distributions in the sprays[Kim et al. 2009], and so too affect their larger scale behaviour through drag and other external influences. Various studies of these interactions characterise these collisions (or more accurately, their outcomes) into three distinct types: coalescence, stretching separation and reflexive separation[Ashgriz and Poo 1990; Estrade et al. 1999; Orme 1997]. We show these collision types in Fig. 1 and describe them as:

- Coalescence (Fig. 1(top)) - as droplets make contact, the tension forces on their now combined surface overcomes any initial kinetic energy acting to separate them and causes the colliding droplets to stably merge and form a single larger droplet.

- Stretching Separation (Fig. 1(middle)) - a grazing collision with lower surface interaction that, instead of coalescing, only forms a temporary fluid ligament connecting the colliding droplets. The droplets continue to move past each other along their initial trajectories which causes the ligament to stretch and break. Drawing mass from the colliding droplets, this ligament can fragment into smaller satellite droplets.

- Reflexive Separation (Fig. 1(bottom)) - an initially coalescing collision with sufficient kinetic energy to cause large internal deformation, oscillation and subsequent breakup, splitting the combined mass into a collection of satellite droplets. Initially, stretching and subsequent fragmentation occurs along the relative trajectory of the colliding droplets, but for increasing colliding kinetic energy, more aggressive, shattering behaviour occurs instead[Pan et al. 2009] (Fig. 5).

It should be noted that in this paper, as in the supporting engineering references, we only consider binary collisions between pairs of droplets, and that larger interactions can be described by a sequence thereof. Furthermore, there does exist another possible outcome, droplet bounce - where droplets are forced apart by a region of high air pressure between them, causing the droplets to seemingly bounce off one-another. However, we do not model this case in this paper, as it depends on a variety of external parameters (shape on impact, surrounding gas density and flow)[Ashgriz and Poo 1990] that we do not consider in this work and more importantly, has lower relevance for larger droplet sizes, such as those we usually consider in graphics.

\section{SIMULATING DROPLETS}

We consider a system of droplet particles under the influence of gravity, drag and inter-droplet interaction. Each particle in our system represents a single droplet and their interactions are simulated using a discrete collision model that recreates the outcomes described above. The model we describe is the first of its kind to use explicit modelling of droplet collisions for computer graphics applications, and in doing so considers the novel combination of the following components to ensure deterministic, visually consistent results.
This model consists of three key stages: binary collision detection, outcome determination and collision resolution.

- For collision detection, we use sphere-moving sphere intersection tests, approximate neighbourhoods and spatially organised particle data to deterministically detect collisions between pairs of droplets in reasonable time.

- The outcome of the collision is then determined using an experimentally-validated, physically-based threshold model[Ashgriz and Poo 1990], which we extend to use a full range of input states.

- Finally, to resolve these collision outcomes, we use a combination of momentum/mass conservation, an existing physically-based fragmentation model[Munnannur and Reitz 2007] and novel non-uniform initial behaviours for satellite droplets.

The existing models we use are chosen as they are well-cited, physically meaningful and show good accordance with experimental results. We will now describe each stage of this model in detail and present the resulting algorithm in Algorithm 1. We begin with the more novel and foreign components of this work - the outcome determination and collision resolution methods.

\section{COLLISION TYPE DETERMINATION}

Suppose we have a collection of colliding droplets, we determine which outcomes (as in Fig. 1) should occur using the binary collision model of Ashgriz and Poo [1990]. Each pairwise collision is characterised using three intrinsic parameters of its colliding state: the Weber number We, the impact parameter $X$ and the droplet size ratio $\delta$.

For the collision between droplets $i$ and $j$ with radii $r_{i} \geq r_{j}$, velocities $\mathbf{u}_{i}, \mathbf{u}_{j}$ and positions $\mathbf{x}_{i}, \mathbf{x}_{j}$ these are defined as follows:

- Weber Number - the ratio of inertial forces to surface tension forces gives the Weber number, We $=\frac{2 \rho r_{j}\left\|\mathbf{u}_{i j}\right\| \|^{2}}{\sigma}$, for relative velocity $\mathbf{u}_{i j}=\mathbf{u}_{j}-\mathbf{u}_{i}$, liquid material density $\rho$ and surface tension coefficient $\sigma$.

- Impact - the shortest distance between the two droplet centres orthogonal to the relative velocity (Fig. 2), normalised by the droplet radii gives the impact, $X=\frac{x}{r_{i}+r_{j}}=$ $\frac{\left\|\mathbf{x}_{i j}-\mathbf{x}_{i j}^{T} \hat{\mathbf{u}} \hat{\mathbf{u}}\right\|}{r_{i}+r_{j}}$, where $x$ is the perpendicular distance between droplet $i$ and the line along $\mathbf{u}_{i j}, \mathbf{x}_{i j}=\mathbf{x}_{j}-\mathbf{x}_{i}$ and $\hat{\mathbf{u}}=\frac{\mathbf{u}_{i j}}{\left\|\mathbf{u}_{i j}\right\|}$.

- Size Ratio - the size ratio of the droplets, $\delta=\frac{r_{j}}{r_{i}}$.

The Weber number is the defining characteristic of a collision, representing the relative energies working to separate and fragment or merge the colliding droplets, with high Weber number collisions favouring separation and break-up due to dominant kinetic energies. The impact and size ratios are used to calculate the volumes (and so masses) and surface areas present in the interaction, determining whether a collision is considered grazing or head-on.

This model then uses comparisons of the opposing kinetic and surface energies present in the collision to define thresholds upon the values of We, $X, \delta$ that classify the appropriate outcome into one of the three types (Fig. 3). To be concise, we give a statement of the final thresholds below but refer the interested reader to the 


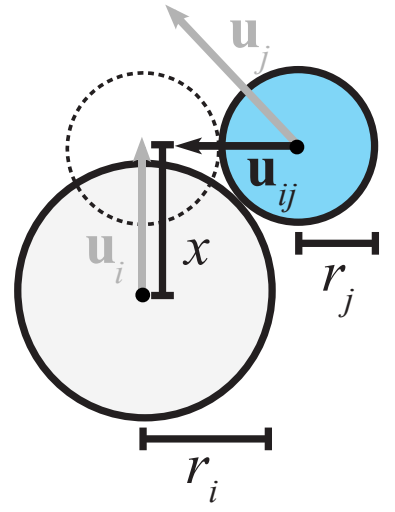

Figure 2: Geometric parameters of a collision between droplets $i$ and $j$. Radii $r_{i}, r_{j}$, relative velocity $\mathbf{u}_{i j}=\mathbf{u}_{j}-\mathbf{u}_{i}$ and $x$ the shortest distance between the droplet centres throughout the collision, assuming linear trajectories.

supplementary material (and Ashgriz and Poo [1990]) for details of their motivation and derivation.

\subsection{Collision type thresholds}

Stretching separation (Fig. 1(middle)) occurs when the kinetic energies of the interacting and non-interacting parts of the droplets are greater than the surface energy of the temporary fluid ligament that would form between them. This energy-based criterion (given in the supplementary material) is reformulated into the following threshold on We such that We $>\mathrm{We}_{\text {stretch }} \rightarrow$ stretching separation for

$$
\mathrm{We}_{\text {stretch }}=\frac{4\left(1+\delta^{3}\right)^{2}\left[3(1+\delta)(1-X)\left(\delta^{3} \phi_{j}+\phi_{i}\right)\right]^{\frac{1}{2}}}{\delta^{2}\left[\left(1+\delta^{3}\right)-\left(1-X^{2}\right)\left(\phi_{j}+\delta^{3} \phi_{i}\right)\right]}
$$

where $\phi_{i}, \phi_{j}$ (Fig. 4) are the fractions of the droplet volumes $V_{i}, V_{j}$ for $i, j$ resp. that interact with the other droplet during the collision. $\phi_{i}, \phi_{j}$ are calculated by sweeping the smaller droplet along the relative velocity and considering the segments/caps of the spheres that overlap (see the supplementary material for an explicit formulation).

Reflexive separation (Fig. 1(bottom)) occurs when the kinetic energy of the deforming combined mass is greater than $\frac{3}{4}$ of the surface energy of the nominal coalesced droplet. This is similarly given by a threshold on We such that $\mathrm{We}>\mathrm{We}_{\text {reflex }} \rightarrow$ reflexive separation for

$$
\mathrm{We}_{\text {reflex }}=\frac{3\left[7\left(1+\delta^{3}\right)^{\frac{2}{3}}-4\left(1+\delta^{2}\right)\right] \delta\left(1+\delta^{3}\right)^{2}}{\left(\delta^{6} \eta_{1}+\eta_{2}\right)}
$$

where $\eta_{1}=2(1-\xi)^{2}\left(1-\xi^{2}\right)^{1 / 2}-1, \eta_{2}=2(\delta-\xi)^{2}\left(\delta^{2}-\xi^{2}\right)^{1 / 2}-\delta^{3}$ for $\xi=0.5 X(1+\delta)$.

If neither of these thresholds are surpassed, the surface energy is sufficient to bring the droplets together to stably merge and the collision is considered a coalescence (Fig. 1(top)) collision.

4.1.1 Considering all collisions. Whilst the thresholds are defined in relative terms and are therefore scale independent (as long as the spherical droplet assumption applies), Ashgriz and Poo [1990] consider only a narrow range of input collision parameters,

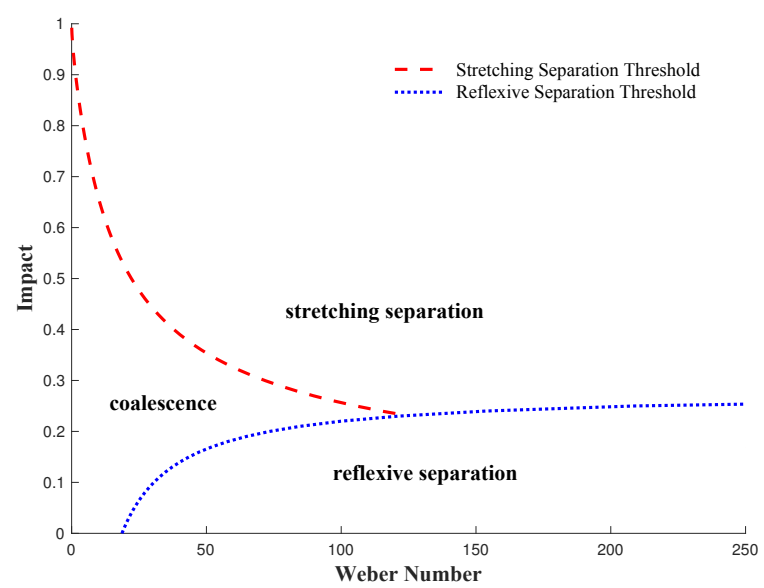

Figure 3: Collision type regions defined by the Weber number, We, and impact, $X$, for a collision between droplets of size ratio, $\delta=1$, using our extended version of the model from Ashgriz and Poo [1990].

$0.5 \leq \delta \leq 1$ and We $\leq 100$. We make some additions to allow the full possible range of inputs that may arise during our simulation. Similar extended models have been used and validated in other works[Kim et al. 2009; Munnannur and Reitz 2007] and we find that the following extensions do not introduce any visible artefacts.

For high velocities or larger droplets (i.e. large $\mathrm{We}$ ), we require handling of collisions that surpass both the reflexive and stretching separation thresholds. Similarly, for lower $\delta$ and $X$, we may find collisions where the smaller droplet is fully interacting, which were not considered in the original work. In the case of both thresholds being surpassed (only occurring for lower values of $X$ ) we assume that reflexive separation takes precedence over stretching separation (Fig. 3) as this behaviour better describes head-on collision. Furthermore, for fully interacting smaller droplets, we cap the original formulation for $\phi_{j}$ to assume full interaction (i.e. $\phi_{j}=1$ ) and calculate the interacting region $\phi_{i}$ using the geometric formulae for spherical segments and caps.

\section{COLLISION RESOLUTION}

Following the determination of the appropriate outcome for each collision, we update our system accordingly to demonstrate these

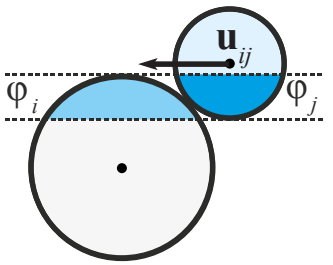

Figure 4: Interaction volumes of droplets $i$ and $j . \phi_{i}, \phi_{j}$ are the fractions of the droplet volumes that are overlapped as droplet $j$ is swept along the velocity $u_{i j}$. 
phenomena. Each outcome type requires handling differently. Coalescence is represented by the merging of droplets whilst the separating collision types require updated radii and velocities alongside the possible emission of additional satellite droplets through fragmentation.

In the following, consider the resolution of the single pairwise collision between droplets $i, j$ from the previous section.

\subsection{Coalescence}

We assume that the larger droplet absorbs the smaller droplet, updating the radius and velocity to conserve mass and momentum and updating the position to the combined centre of mass.

\subsection{Separation and fragmentation}

Although arising from differing interactions, both separating collision outcomes display similar behaviour and as such are modelled in a similar manner.

Firstly we update the colliding droplet velocities to be

$$
\tilde{\mathbf{u}}_{k}=\frac{r_{k}^{3} \mathbf{u}_{k}+r_{l}^{3} \mathbf{u}_{l}-r_{l}^{3}\left(\mathbf{u}_{k}-\mathbf{u}_{l}\right) z}{r_{k}^{3}+r_{l}^{3}}
$$

for $k, l=i, j$ where $z=\frac{X-\sqrt{\frac{2.4 f(\delta)}{\mathrm{We}}}}{1-\sqrt{\frac{2.4 f(\delta)}{\mathrm{We}}}}, f(\delta)=\delta^{-3}-2.4 \delta^{-2}+2.7 \delta^{-1}$ $(z \geq 0)$ for stretching separation and $z=\sqrt{1-\frac{W_{\text {reflex }}}{W e}}$ for reflexive separation (taken from O'Rourke [1981] and Tennison et al. [1998] resp.).

Then, to calculate possible fragmentation in these outcomes we use the method from Munnannur and Reitz [2007]. This considers fragmentation during collision to be the result of the break-up of a temporary cylindrical liquid ligament due to the Plateau-Rayleigh instability[Rayleigh 1878]. Note that although this method does not properly describe the mechanisms behind shattering reflexive separations[Pan et al. 2009] we later demonstrate the ability to approximate similar results using our improved satellite creation (Fig. 5).

Munnannur and Reitz [2007] calculate the break-up of this ligament into satellite droplets by first calculating of the volume of liquid involved. Then, knowing the stretching velocity $\mathbf{u}_{i j}$, give a subsequent equation for the ligament radius at breakup, and in turn, the radius of any resulting satellite droplets. The following sections describe these calculations.

5.2.1 Ligament volume. For stretching separation, the liquid ligament forms between the two droplets as they graze past oneanother. This volume, $V_{\text {lig }}$, is assumed to be equal to some fraction of the total interaction volume of the droplets, dependent on the magnitude of the opposing surface, stretching and viscous dissipation energies (as used by Ashgriz and Poo [1990] to calculate the collision type thresholds) in the collision s.t.:

$$
V_{\text {lig }}=C_{\text {sep }}\left(V_{\text {interact }, i}+V_{\text {interact }, j}\right)
$$

where $V_{k}=\frac{4}{3} \pi r_{k}^{3}$ and $V_{\text {interact }, k}=V_{k} \phi_{k}$ for $k=i, j$ and $\phi_{k}$ as in Fig. (4) and $C_{s e p}<1$, known as the separation volume coefficient, is

$$
C_{\text {sep }}=\frac{\mathbf{E}_{\text {stretch }}-\mathbf{E}_{\text {surface }}-\mathbf{E}_{\text {dissip }}}{\mathbf{E}_{\text {stretch }}+\mathbf{E}_{\text {surface }}+\mathbf{E}_{\text {dissip }}}
$$

where

$$
\begin{gathered}
\mathbf{E}_{\text {stretch }}=\frac{1}{2} \rho\left\|\mathbf{u}_{i j}\right\|^{2} V_{i}\left(\frac{\delta^{3}}{\left(1+\delta^{3}\right)^{2}}\right)\left[\left(1+\delta^{3}\right)-\left(1-X^{2}\right)\left(\phi_{j}+\delta^{3} \phi_{i}\right)\right] \\
\mathbf{E}_{\text {surface }}=2 \sigma\left[\pi V_{i} r_{i} \tau\left(\phi_{i}+\delta^{3} \phi_{j}\right)\right]^{\frac{1}{2}}
\end{gathered}
$$

for $\tau=(1-X)(1+\delta)$ and $\mathbf{E}_{\text {dissip }}$ is $30 \%$ of the initial kinetic energy of the colliding droplets.

For reflexive separations, this ligament is considered to be the entire temporarily merged mass s.t. $V_{\text {lig }}=V_{i}+V_{j}$, which is instead stretching due to internally-driven deformations along the relative velocity (known as prolate phase oscillation).

5.2.2 Ligament instability. The radius at breakup $r_{b u}$ of the cylindrical liquid ligament (assuming initial length equal to its radius $r_{0}$ ) extending with velocity $\mathbf{u}_{i j}$, is then given as the solution to:

$$
\beta \mathrm{We}_{0}^{\frac{1}{2}}\left(\frac{r_{b u}}{r_{0}}\right)^{\frac{7}{2}}+\left(\frac{r_{b u}}{r_{0}}\right)^{2}-1=0
$$

where $\beta=\frac{3}{4 \sqrt{2}}\left(k_{1} k_{2}\right), k_{1}=11.5, k_{2}=0.45$ (using updated constants from Kim et al. [2009]) and $\mathrm{We}_{0}=2 r_{0} \frac{\rho}{\sigma}\left\|\mathbf{u}_{i j}\right\|^{2}$.

Finally, assuming that this breakup occurs due to the disturbance of the ligament that has the maximum growth rate and that the volume of each satellite is equal to the cylinder of length corresponding to the wavelength of this disturbance, Munnannur and Reitz [2007](citing Georjon and Reitz [1999]) give the radius of each satellite as $r_{\text {sat }}=1.89 r_{b u}$.

5.2.3 Number of satellites. If $V_{\text {lig }}>0$ we use the above calculations to find $r_{s a t}$ and we are then able to calculate the number of possible spherical satellites created from the ligament breakup, $n_{\text {sat }}$, using mass conservation. For stretching separation, we then emit $n_{\text {sat }}$ satellites into our system (using the rules below) with any remaining mass returning to the parent droplets. For reflexive separation if $n_{\text {sat }}<3$, no satellite droplets are created (instead our initial droplets are re-formed) and we make no changes to the incident droplet radii post-collision, otherwise these radii are updated to $r_{\text {sat }}$ alongside the emission of the additional $n_{\text {sat }}-2$ satellites.

\subsection{Satellite creation}

Previous applications of fragmenting models[Kim et al. 2009; Ko and Ryou 2005; Munnannur and Reitz 2007] have assumed simplified criteria for satellite creation, merging all satellites from a collision into a single 'parcel' and initialising them with entirely uniform values. To allow satellites to join our system as otherwise normal droplets, we cannot use these simplifications. Whilst we continue to assume that the satellites are of uniform radii due to the cylindrical nature of the ligament, we emit the satellites evenly along this nominal ligament with velocities that replicate the stretching behaviour causing its break-up. 


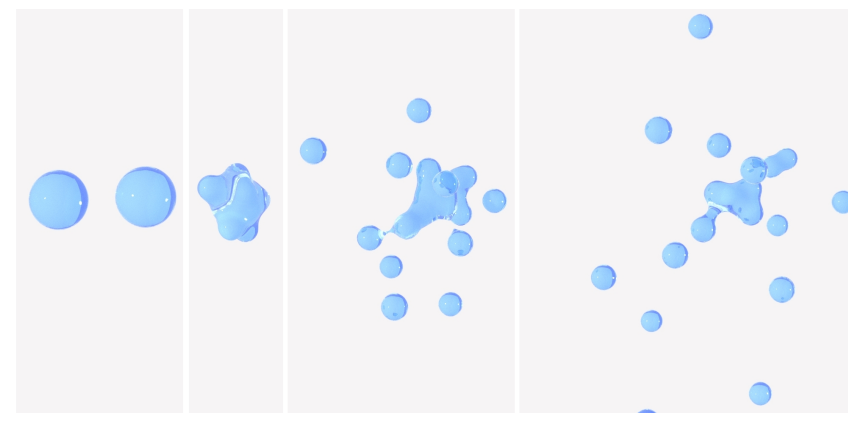

Figure 5: Shattering reflexive separation (left to right), greatly increased kinetic energy version of reflexive separation seen in Figure 1, creating many satellites with perturbed satellite velocities (Section 5.3.1).

The velocities are calculated using momentum conservation with a weighted bias to their closest parent droplet, such that for satellite $n=1, \ldots n_{\text {sat }}$, we have:

$$
\mathbf{u}_{n}=\frac{V_{\text {lig }, i}}{V_{\text {lig }}} \tilde{\mathbf{u}}_{i}+\frac{V_{\text {lig, }, j}}{V_{\text {lig }}}\left(\tilde{\mathbf{u}}_{j}+\left(1-\frac{2 n}{n_{\text {sat }}+1}\right) \tilde{\mathbf{u}}_{i j}\right)
$$

with $\tilde{\mathbf{u}}_{k}$ for $k=i, j$ the post-collision velocity of the parents (Equation 3$), V_{\text {lig, } k}$ for $k=i, j$ the contribution of droplet $k$ to the ligament volume and satellite $n_{\text {sat }}$ is that closest to droplet $j$.

5.3.1 Perturbation and shattering. When droplets collide at high energy, the interacting droplet mass undergoes large deformations and oscillations, perturbing the velocities of the satellites that are created as a result. As the fragmentation model we use provides no information on torque, to approximate this behaviour, we add a random rotation to the velocity of the satellites. We apply a rotation $R_{n}$ to each satellite (including updated colliding droplets in reflexive separations) such that their final velocity is given by $\tilde{\mathbf{u}}_{n}=R_{n} \mathbf{u}_{n}$. The magnitude of this rotation is a uniformly sampled value in $\alpha=\left[0, \eta n_{\text {sat }}\right]$ where $\eta$ defines a user parameter to define the scale of the rotational variation introduced by these deformations, and the perturbation increases with the number of satellites being created. We further scale $R_{n}$ by $(1-\alpha)^{2}$ such that larger perturbations also dissipate more energy. In this way, for extreme cases, we are also able to approximate the behaviour of reflexive separating collisions as they tend to shattering[Pan et al. 2009] (Fig. 5).

\section{BINARY COLLISION DETECTION}

In order to use the above droplet collision model in our particle system, we require a method to detect these pairwise droplet collisions. As the resolution of each collision alters the system, i.e. the trajectories, sizes and numbers of droplets, to avoid costly iterative detection, resolution and advancement of the system within a single timestep $\Delta t$, we choose to constrain each droplet to at most one resolvable collision per timestep. We then use a deterministic, trajectory-based scheme that combines Taskiran and Ergeneman [2014]'s use of sphere-moving sphere intersection tests with a pairing mechanism inspired by Ando et al. [2012]. This is able to find all possible collisions and results in a set of collision pairs where each particle is included at most once.

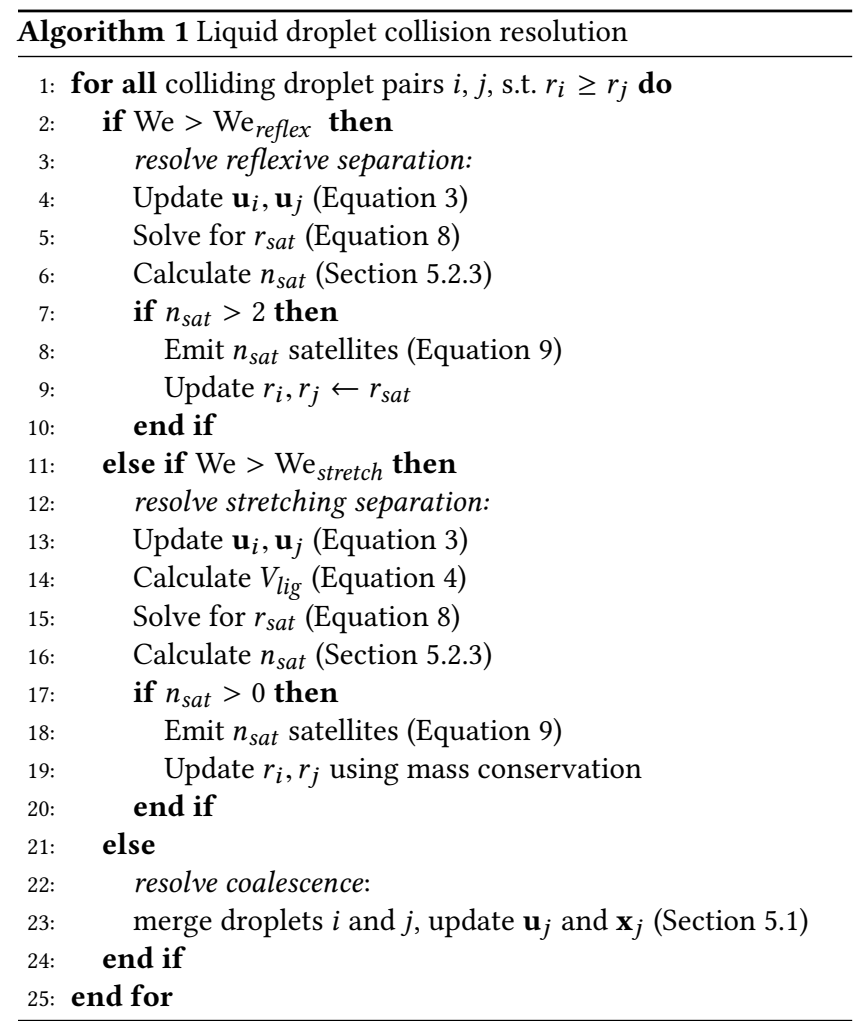

For each pair of particles $i, j$, sphere-moving sphere collision detection[Taskiran and Ergeneman 2014] gives the times of initial collision and separation (assuming linear trajectories along $\mathbf{u}_{i}, \mathbf{u}_{j}$ ) as $t^{ \pm}$, the roots of the polynomial:

$$
\left\|\mathbf{x}_{i j}\right\|^{2}+2\left(\mathbf{u}_{i j} \mathbf{x}_{i j}\right) t+\left\|\mathbf{u}_{i j}\right\|^{2} t^{2}=\left(r_{i}+r_{j}\right)^{2}
$$

Each particle calculates and stores a reference to the corresponding particle with which it has the lowest initial collision time, i.e. lower root $t^{-}$. This is followed by a subsequent pairing step, as in the particle merging scheme of Ando et al. [2012], to check each particle and its preferred collision pair. If both particles refer to the other and their collision time is within the substep $\Delta t$, a binary collision is successfully found and the pair are marked and removed from the next iteration of detection.

To avoid checking each particle against every other particle we define the neighbourhood of all possible collision neighbours for $i$ in $\Delta t$ as:

$$
\begin{aligned}
N_{\text {trajectory }, i}=\{\text { particles } j \text { s.t. } & \left\|\mathbf{x}_{i}-\mathbf{x}_{j}\right\|< \\
& \left.r_{i}+r_{\text {max }}+\Delta t\left(\left\|\mathbf{u}_{i}\right\|+u_{\text {max }}\right)\right\}
\end{aligned}
$$

where $r_{\max }$ and $u_{\max }$ are the maximum radii and speed of any droplets in the system.

This method is able to find all possible collisions that can occur, even considering high velocity, large timesteps or very small particles. However, the size of the neighbourhood $N_{\text {trajectory, } i}$ can make this very costly. In practice, we have found that it is sufficient to consider an approximation of this neighbourhood, only allowing 
collisions at the substep interval, denoted $N_{\text {overlap, } i}$, given by

$$
N_{\text {overlap }, i}=\left\{\text { particles } j \text { s.t. }\left\|\mathbf{x}_{i}-\mathbf{x}_{j}\right\|<r_{i}+r_{\max }\right\}
$$

For this neighbourhood, the collision detection test above is still used to calculate the initial time of collision, with the lowest continuing to take precendence. Using the iterative checking mechanism, we can combine these neighbourhoods when considering fast moving, sparse particle sets. For example, using a number of iterations of detection within $N_{\text {overlap, } i}$ before testing $N_{\text {trajectory, } i}$ can help to reduce the number of droplets considered when using the more costly larger neighbourhood, if required. However, for most situations we have found that even at low iteration counts $(<3)$ we are able to find a significant number of unique collision pairs using only the reduced neighbourhood, for reasonable timestep sizes.

\section{DRAG}

Another important element in recreating droplet spray motion is the inclusion of a physically plausible drag force. Given our system of variable-radii droplet particles, we are able to replicate the realistic dependence of the drag force upon droplet size.

Assuming a spherical droplet, radius $r$, moving through the air at velocity $\mathbf{u}$, the usual Newton drag force on the droplet is[Sirignano 2010]:

$$
\mathbf{F}_{\text {Newton }}=\frac{1}{2} C_{D} \rho_{\text {air }} \pi r^{2} \Delta \mathbf{u}^{2}
$$

where $\Delta \mathbf{u}^{2}=\|\Delta \mathbf{u}\| \Delta \mathbf{u}$ for $\Delta \mathbf{u}=\mathbf{u}_{\text {air }}-\mathbf{u}, C_{D}$ is the drag coefficient and $\mathbf{u}_{\text {air }}, \rho_{\text {air }}$ are the velocity and density of the surrounding air. However, for smaller-scale droplets, where viscous forces in the air are more dominant, this should instead be of the form of Stokes' drag given by:

$$
\mathbf{F}_{\text {Stokes }}=6 \pi \rho_{\text {air }} v_{\text {air }} r \Delta \mathbf{u}
$$

where $v_{\text {air }}$ is the viscosity of the surrounding air.

To allow application of these drag forces within our particle system we assume that the magnitude of the background air velocity is negligable compared to the droplet velocity and replace the $\Delta \mathbf{u}$ term with $-\mathbf{u}$. Then for simplicity, combine all other terms (including terms from the mass, $m$ ) into a single user parameter $\alpha>0$ (as in Song et al. [2005]) and the choice of exponent $\sigma$ such that the velocity update is as follows:

$$
\frac{d \mathbf{u}}{d t}=-\frac{\alpha}{r^{\sigma}} \mathbf{u}^{3-\sigma}
$$

where $\sigma \in 1,2$ such that the drag equation mimics either the Newton drag (Equation 13) or Stokes drag (Equation 14) for larger and smaller scale simulations respectively. As in Mihalef et al. [2009], these could also be combined with an interpolation scheme but we have not found the need to do so.

\section{IMPLEMENTATION}

This model has been implemented into a simple particle system built using OpenVDB Points[Museth et al. 2015] as a plugin for Houdini[Side Effects 2016]. All of the components described in this paper are implemented as multi-threaded operations, parallelised over the leaves of the OpenVDB Points grid.
Table 1: Suggested values for the parameters considering material properties of water/droplets. The relative nature of the collision parameters makes these somewhat flexible and allows artistic control through their modification e.g. modelling the approximate behaviour of larger 'droplets' than those in reality (Fig. 8)

\begin{tabular}{lll}
\hline Name & Description & Default \\
\hline$\sigma$ & surface tension coefficient $(\mathrm{N} / \mathrm{m})$ & 0.072 \\
$\rho$ & liquid density $\left(\mathrm{kg} / \mathrm{m}^{3}\right)$ & 997.044 \\
$\alpha$ & drag coefficient & 0.0001 \\
$\Delta t$ & timestep size $(\mathrm{s})$ & $1 / 24$ \\
$r_{\text {max }}$ & maximum droplet radius $(\mathrm{mm})$ & $10-100$ \\
$r_{\text {min }}$ & minimum droplet radius $(\mathrm{mm})$ & $0.05-0.5$ \\
$\eta$ & satellite velocity perturbation & 0.01 \\
$t_{\text {delay }}$ & minimum time between collisions $(\mathrm{s})$ & $1 / 24$ \\
$n_{\text {max }}$ & maximum satellites created per collision & 5 \\
$r_{0}$ & initial droplet radius $(\mathrm{mm})$ & 1 \\
\hline
\end{tabular}

Solving Equation (8). : We use bracket_and_solve_root in the Boost library[Schäling 2011] with an initial guess of the solution to the 3rd order polynomial

$$
\beta \mathrm{We}_{0}^{\frac{1}{2}}\left(\frac{r_{b u}}{r_{0}}\right)^{3}+\left(\frac{r_{b u}}{r_{0}}\right)^{2}-1=0
$$

Neighbourhood search: The inherent spatial organisation of the point data structure allows a fast and easy implementation of the collision detection system that we describe in this paper, without the requirement for any other acceleration structures.

Time of resolution: Collisions are resolved at the impact time, i.e. $t_{X}=t^{-}+\frac{t^{+}-t^{-}}{2}$, when the droplets are most overlapping, however we often cap this to some max value, i.e. $\Delta t$, for collisions with very large $t_{X}$. Droplets are advanced to this time, resolved and then back-advected with their post-collision velocities through $t_{X}$, such that all droplets are in the correct positions accounting for their mid-timestep velocity change after they are advected as normal through $\Delta t$.

Rest between collisions: Each droplet, once collided is deactivated from collisions for a few timesteps. This allows ligament breakup to fully resolve and satellites to separate, avoiding over detection of collisions and excessive merging in slower moving scenes.

Drag magnitude: This is clamped by the input velocity so that it does not cause direction changes.

Droplet sizes: To avoid over-merging of droplets and/or the introduction of very small droplets we clamp the droplet sizes to a user defined range. We skip any coalescence/fragmentation that would produce droplets outside this range but continue to allow large droplets to fragment, small droplets to coalesce etc.

Surfacing: For close-up examples, to better demonstrate ligament stretching and breakup using our particles we rasterize particles into a VDB level-set, apply smoothing operations (as described by Museth [2014]) and subsequently convert to a mesh for rendering, all using the existing VDB operators in Houdini[Side Effects 2016]. 
Table 2: Simulation times for results shown. The complexity of the operations, including the emission and deletion of particles, and widely varying particle count arising from interactions means results can differ considerably. All simulations performed with two 8-core 3.10Ghz Intel Xeon CPUs and 64GB RAM.

\begin{tabular}{lllll}
\hline Example & Frame time w/o collision & with collision & Final \# particles w/o collision & with collision \\
\hline Fig. 7 (our model) & $0.025 \mathrm{~s}$ & $0.028 \mathrm{~s}$ & 200 & 139 \\
Fig. 7 (FLIP) & $0.055 \mathrm{~s}$ & N/A & 200 & N/A \\
Fig. 7 (SPH) & $0.006 \mathrm{~s}$ & N/A & 200 & N/A \\
Fig. 7 (RBD) & $0.006 \mathrm{~s}$ & N/A & 200 & N/A \\
Fig. 8 (second from top) & $0.101 \mathrm{~s}$ & $0.277 \mathrm{~s}$ & 111,532 & 181,766 \\
Fig. 8 (second from bottom) & $0.101 \mathrm{~s}$ & $0.136 \mathrm{~s}$ & 111,532 & 57,846 \\
Fig. 8 (bottom) & $0.101 \mathrm{~s}$ & $0.099 \mathrm{~s}$ & 111,532 & 22,265 \\
Fig. 9 & $1.35 \mathrm{~s}$ & $1.89 \mathrm{~s}$ & $3,235,474$ & $1,332,316$ \\
\hline
\end{tabular}

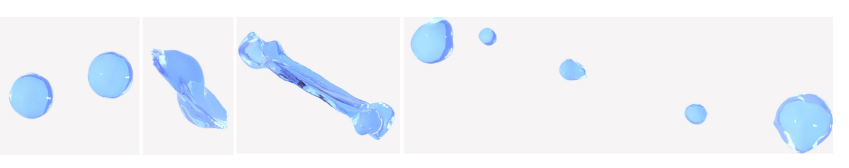

Figure 6: Stretching separation using FLIP simulation in Houdini. Requires 18k particles to replicate behaviour that we simulate with a single pair of particles (Fig. 1(middle)).

\section{RESULTS AND DISCUSSION}

By introducing these interactions into a simple particle system, we are able to plausibly and efficiently model large systems of water droplets. We first demonstrate the simplest, fundamental case of a single binary droplet collision in Fig. 1, showing how our model approximates ligament formation and handles the topology changes that occur during the interactions described in Section 2.2. We compare our stretching separation result to a recreation using Houdini's FLIP liquid solver in Fig. 6, which we found requires a system of at least $18 \mathrm{k}$ particles to demonstrate the same stretching/fragmenting phenomena (albeit with added qualities eg. surface deformation).

In Fig. 7, we show a close-up of a small system of droplets to further demonstrate the interactions that occur. Comparisons are given to the attempted use of FLIP, SPH and rigid body solvers (native to Houdini) to create similar interactions for the same input particle set. The coalescence and fragmentation that we model create levels of detail and realistic liquid features (varying size droplets, stretching of ligaments) that are not created by other methods, demonstrating their lack of a meaningful one-to-one mapping between droplet and particle. This result also demonstrates the potential for this method to be used for fast simulation of small-scale droplet interactions, however further additions would be required to overcome the spherical uniformity of the particles and merging behaviours at this scale, such as for bubbles in Patkar et al. [2013].

Following this, we demonstrate the effect of our model on largerscale spray effects that are often simulated with ballistic particle systems. To best illuminate the specific effects of our model we restrict these examples to an otherwise purely ballistic system. However, in practice, our model can easily be combined with other available tools used with these systems such as noise fields, droplet age and other external forces. In Fig. 8 we simulate a geyser-like vertical jet of liquid droplets. In this example, the introduction of these mechanisms for droplet merging and splitting increases visual

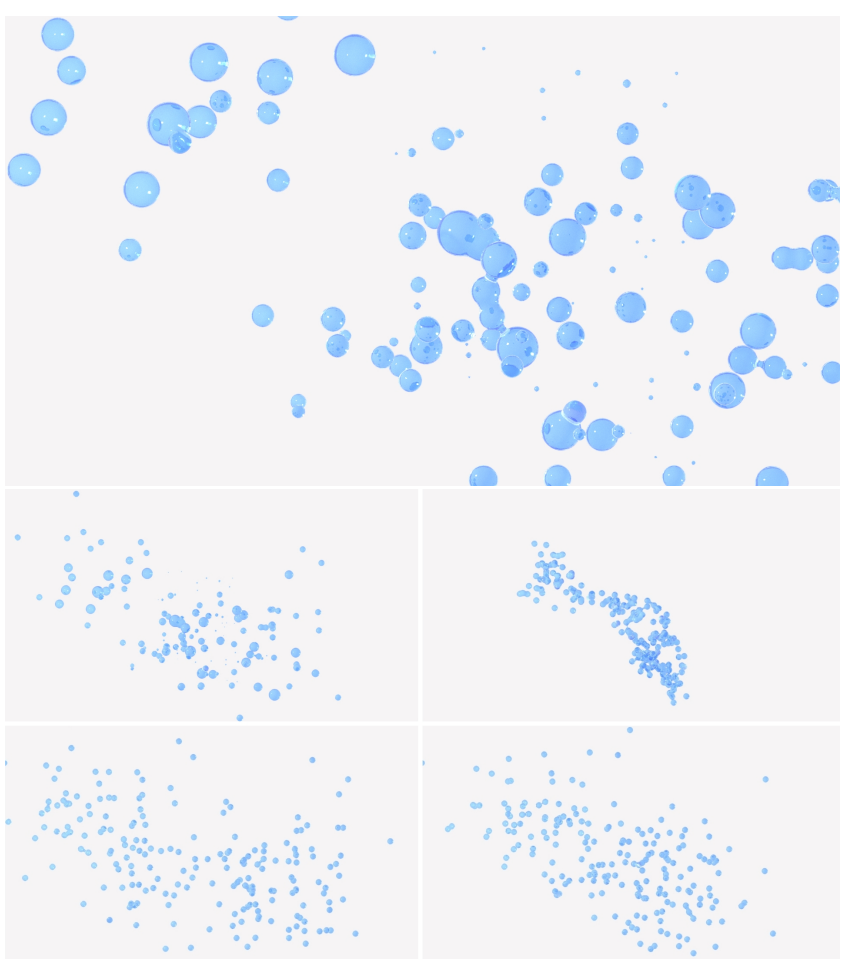

Figure 7: Small-scale system of droplet interactions: closeup using our droplet collision model (top); zoomed out view using our model (middle left), using FLIP solver (middle right), SPH solver (bottom left) and rigid body (RBD) solver (bottom right). While FLIP does create larger-scale motions, other systems are unable to create realistic smallscale droplet characteristics, e.g. size variation, ligament formation and fragmentation, at low particle density.

quality and reduces artefacts seen in the ballistic particle equivalent, here from a poorly constructed emitter creating uniformity that persists due to lack of any interaction. The other panels of this figure further demonstrate the influence of parameter variation on our model, controlling the tendency to coalesce/fragment and varying the resulting droplet distribution between an finer spray and fewer, larger droplets. 


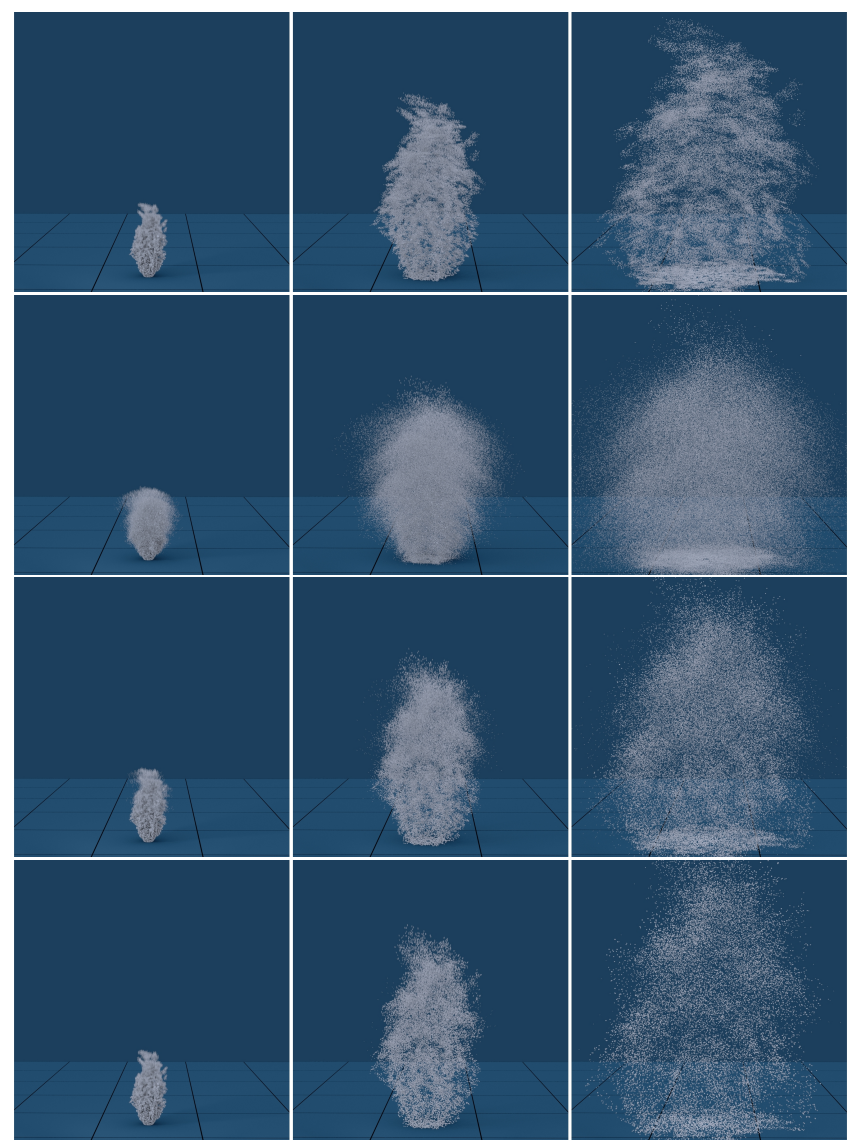

Figure 8: Geyser simulation (left to right): purely ballistic particle system - top, our model with reduced surface tension $(\times 0.1)$ - second from top, our model - second from bottom, our model with increased surface tension $(\times 10)$ - bottom. Low surface tension interactions cause many satellites to be emitted and create a finer spray effect, whilst high surface tension tends to coalescence and causes system to tend to fewer, larger droplets.

Finally, we use this model to approximate very fine-scale spray effects, considering millions of particles in the fountain example of Fig. 9. Here, uniform radius particles are initially perturbed by a small amount of curl-noise[Bridson et al. 2007] to vary the flow and allow interactions. Whilst largely dominated by coalescence, the other interactions in this example allow the system to maintain a range of droplet sizes which, in combination with the size-dependent drag force, break-up the uniformity of the flow and create a realistic fountain effect.

These larger simulation examples demonstrate that even in scenarios where droplet size and individual interactions are not as easy to directly recognise, their effect on the droplet distribution continues to improve the quality of the results, adding an increased sense of realistic motion and scale.

Limitations: Modelling interactions between particles increases simulation time over simple ballistic particle tracking, in particular

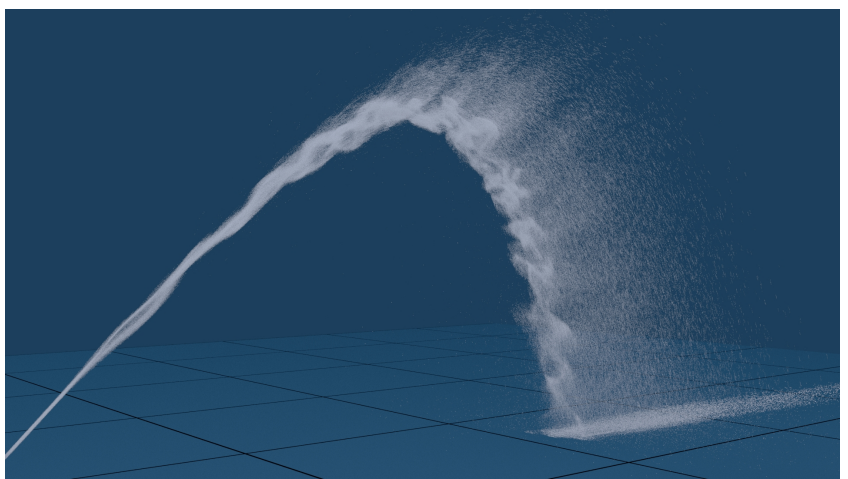

Figure 9: Fountain-like jet (1.3m particles): Interactions create a varied droplet size distribution and therefore drag strength, which causes larger-scale break-up of the flow.

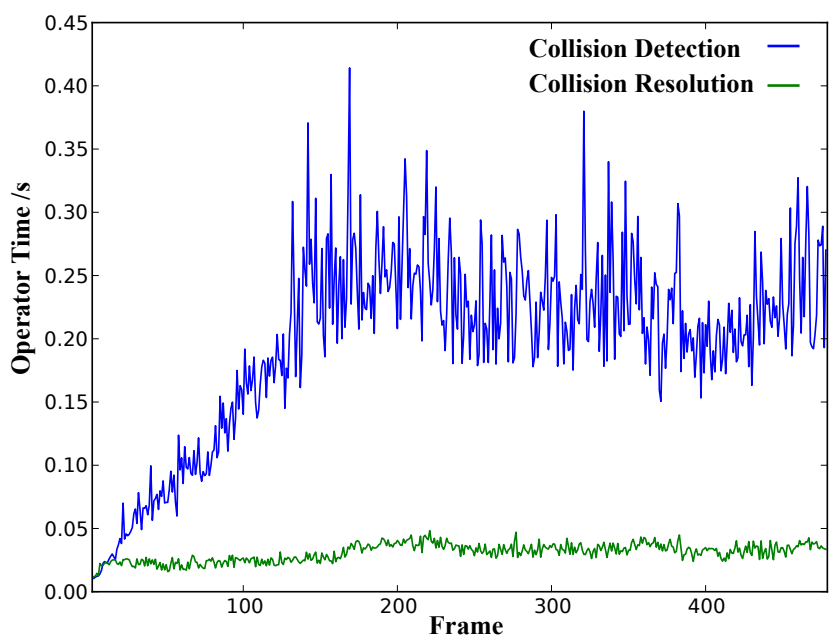

Figure 10: Times of collision detection and collision resolution (incl. outcome determination and satellite emission) operations for simulation in Fig. 9. 480 frames with 2 substeps per frame, $\Delta t=1 / 96$.

due to the collision detection stage (Fig. 10). We find that the approximate neighbourhood method, the use of spatially organised particle data, and often reduced particle count brings this cost down to a manageable level for the increase in quality that is gained (Table 2), but further improvements could be made. Furthermore, the discrete threshold model and fragmentation calculation that we use are somewhat complicated (albeit moreso in understanding than usability or computational complexity). We chose these methods as they are physically meaningful and shown to have good accordance to real behaviour, but there may be simplifications possible in the future. Finally, as this model defines droplet-like interactions specifically, in areas where the droplet approximation breaks down, i.e. very dense sprays/bodies of liquid, we are unable to capture larger-scale fluid-like motions. This could be overcome by appropriate coupling of this method to larger-scale fluid motion, but we leave this open research question to future work. 


\section{CONCLUSION}

In this paper, we have introduced a model for physically-based interaction to be incorporated into particle systems of liquid droplets and sprays. Our model is the first of its kind to consider the visible impact of the various separating droplet phenomena that occur in reality, and in doing so presents a novel physically-based method to define a complete system of merging and splitting phenomena between droplets. The results that we provide demonstrate an increased sense of scale and physicality from the inclusion of these interactions, creating varied and evolving droplet size distributions and increased small-scale details from fragmentation. Our model recreates plausible behaviours of droplets and sprays on both small and large scales, capturing localised interaction in scenarios where existing fluid techiques fail and helping the creation of larger-scale effects when combined with our size-dependent drag force.

By instilling missing physical characteristics into our system, we have allowed more realistic phenomena to be recreated and we expect that similar physically-motivated approaches would also be useful for other secondary fluid phenomena. We forsee the logical extension of this work to be the coupling of our small-scale interactions with larger-scale fluid behaviours in the creation of a more comprehensive splash/spray system. Other work could also look to build on this model to incorporate missing physical behaviours (i.e. surface deformations, continous merging) to allow fast simulation of close-up droplet effects.

\section{ACKNOWLEDGEMENT}

This research was supported by EPSRC grant EP/G037736/1. We would also like to thank James Bird, Matt Warner, Nick Avramoussis, Todd Keeler, Francisco Gochez and Dan Bailey on the FX R\&D team at Double Negative for their input and support.

\section{REFERENCES}

R. Ando, N. Thürey, and R. Tsuruno. 2012. Preserving fluid sheets with adaptively sampled anisotropic particles. IEEE Transactions on Visualization and Computer Graphics 18, 8 (2012), 1202-1214.

N. Ashgriz. 2011. Handbook of Atomization and Sprays: Theory and Applications. Springer Science \& Business Media.

N. Ashgriz and J.Y. Poo. 1990. Coalescence and separation in binary collisions of liquid drops. Fournal of Fluid Mechanics 221 (1990), 183-204.

O. Azencot, O. Vantzos, M. Wardetzky, M. Rumpf, and M. Ben-Chen. 2015. Functional thin films on surfaces. In Proceedings of the 2015 ACM SIGGRAPH/Eurographics Symposium on Computer Animation. 137-146.

P.R. Brazier-Smith, S.G. Jennings, and J. Latham. 1972. The interaction of falling water drops: coalescence. In Proceedings of the Royal Society of London A: Mathematical, Physical and Engineering Sciences, Vol. 326. 393-408.

R. Bridson, J. Houriham, and M. Nordenstam. 2007. Curl-noise for procedural fluid flow. ACM Transactions on Graphics (TOG) 26, 3 (2007), 46.

O. Busaryev, T.K. Dey, H. Wang, and Z. Ren. 2012. Animating bubble interactions in a liquid foam. ACM Transactions on Graphics (TOG) 31, 4 (2012), 63.

N. Chentanez, M. Müller, and T.Y. Kim. 2015. Coupling 3D Eulerian, heightfield and particle methods for interactive simulation of large scale liquid phenomena. IEEE Transactions on Visualization and Computer Graphics 21, 10 (2015), 1116-1128.

F. Da, D. Hahn, C. Batty, C. Wojtan, and E. Grinspun. 2016. Surface-only liquids. ACM Transactions on Graphics (TOG) 35, 4 (2016), 78.

J.P. Estrade, H. Carentz, G. Lavergne, and Y. Biscos. 1999. Experimental investigation of dynamic binary collision of ethanol droplets-a model for droplet coalescence and bouncing. International fournal of Heat and Fluid Flow 20, 5 (1999), 486-491.

E. Froemling, T. Goktekin, and D. Peachey. 2007. Simulating whitewater rapids in ratatouille. In ACM SIGGRAPH 2007 Sketches. 68.

T.L. Georjon and R.D. Reitz. 1999. A drop-shattering collision model for multidimensional spray computations. Atomization and Sprays 9, 3 (1999).

M. Ihmsen, N. Akinci, G. Akinci, and M. Teschner. 2012. Unified spray, foam and air bubbles for particle-based fluids. The Visual Computer 28, 6-8 (2012), 669-677.
M. Ihmsen, J. Orthmann, B. Solenthaler, A. Kolb, and M. Teschner. 2014. SPH Fluids in Computer Graphics. In Eurographics 2014 - State of the Art Reports.

J. Iversen and R. Sakaguchi. 2004. Growing up with fluid simulation on The Day After Tomorrow. In ACM SIGGRAPH 2004 Sketches. 142.

J. Kim, D. Cha, B. Chang, B. Koo, and I. Ihm. 2006. Practical animation of turbulent splashing water. In Proceedings of the 2006 ACM SIGGRAPH/Eurographics Symposium on Computer Animation. 335-344.

S. Kim, D.J. Lee, and C.S. Lee. 2009. Modeling of binary droplet collisions for application to inter-impingement sprays. International fournal of Multiphase Flow 35, 6 (2009), 533-549.

G.H. Ko and H.S. Ryou. 2005. Modeling of droplet collision-induced breakup process. International fournal of Multiphase Flow 31, 6 (2005), 723-738.

M. Kurtz and G. Duda. 2002. Foamy creatures: Digital Domain wrangles whitewater for Lord of the Rings. In ACM SIGGRAPH 2002 Conference Abstracts and Applications. 186-186.

F. Losasso, J. Talton, N. Kwatra, and R. Fedkiw. 2008. Two-way coupled SPH and particle level set fluid simulation. IEEE Transactions on Visualization and Computer Graphics 14, 4 (2008), 797-804.

M. Macklin and M. Müller. 2013. Position based fluids. ACM Transactions on Graphics (TOG) 32, 4 (2013), 104.

V. Mihalef, D. Metaxas, and M. Sussman. 2009. Simulation of two-phase flow with sub-scale droplet and bubble effects. In Computer Graphics Forum, Vol. 28. 229-238.

A. Munnannur and R.D Reitz. 2007. A new predictive model for fragmenting and non-fragmenting binary droplet collisions. International fournal of Multiphase Flow 33, 8 (2007), 873-896.

K. Museth. 2014. A flexible image processing approach to the surfacing of particlebased fluid animation (invited talk). In Mathematical Progress in Expressive Image Synthesis I. 81-84.

K. Museth, D. Bailey, J. Budsberg, J. Lynch, and A. Pearce. 2015. OpenVDB. In ACM SIGGRAPH 2015 Courses. 20.

M.B. Nielsen and O. Østerby. 2013. A two-continua approach to Eulerian simulation of water spray. ACM Transactions on Graphics (TOG) 32, 4 (2013), 67.

M. Orme. 1997. Experiments on droplet collisions, bounce, coalescence and disruption. Progress in Energy and Combustion Science 23, 1 (1997), 65-79.

P.J. O'Rourke. 1981. Collective drop effects on vaporizing liquid sprays. Technical Report. Los Alamos National Lab., NM (USA).

K.L. Pan, P.C. Chou, and Y.J. Tseng. 2009. Binary droplet collision at high Weber number. Physical Review E 80, 3 (2009), 036301.

S. Patkar, M. Aanjaneya, D. Karpman, and R. Fedkiw. 2013. A hybrid LagrangianEulerian formulation for bubble generation and dynamics. In Proceedings of the 2013 ACM SIGGRAPH/Eurographics Symposium on Computer Animation. 105-114.

L. Rayleigh. 1878. On the Instability of Jets. Proceedings of the London Mathematical Society 1, 1 (1878), 4-13.

B. Schäling. 2011. The Boost C++ libraries. Boris Schäling.

Side Effects. 2016. Houdini. (2016). https://sidefx.com

W. A. Sirignano. 2010. Fluid Dynamics and Transport of Droplets and Sprays. Cambridge University Press.

O.Y. Song, H. Shin, and H.S. Ko. 2005. Stable but nondissipative water. ACM Transactions on Graphics (TOG) 24, 1 (2005), 81-97.

T. Takahashi, H. Fujii, A. Kunimatsu, K. Hiwada, T. Saito, K. Tanaka, and H. Ueki. 2003. Realistic animation of fluid with splash and foam. In Computer Graphics Forum, Vol. 22. 391-400.

O.O. Taskiran and M. Ergeneman. 2014. Trajectory based droplet collision model for spray modeling. Fuel 115 (2014), 896-900.

P.J. Tennison, T.L. Georjon, P.V. Farrell, and R.D. Reitz. 1998. An experimental and numerical study of sprays from a common rail injection system for use in an HSDI diesel engine. Technical Report. SAE Technical Paper.

H. Wang, P.J. Mucha, and G. Turk. 2005. Water drops on surfaces. In ACM Transactions on Graphics (TOG), Vol. 24. 921-929.

L. Yang, S. Li, A. Hao, and H. Qin. 2014. Hybrid Particle-grid Modeling for Multi-scale Droplet/Spray Simulation. In Computer Graphics Forum, Vol. 33. 199-208. 